\title{
Comment on "Reversal of trend of biomass burning in the Amazon" by Ilan Koren, Lorraine A. Remer, and Karla Longo
}

\author{
Wilfrid Schroeder \\ Earth System Science Interdisciplinary Center, University of Maryland, \\ College Park, Maryland, USA
}

\section{Louis Giglio}

Science Systems and Applications Inc., Lanham, Maryland, USA

\section{José A. Aravéquia}

Centro de Previsão do Tempo e Estudos Climáticos,

Cachoeira Paulista, Brazil

Received 22 August 2008; revised 12 November 2008; accepted 14 November 2008; published 6 February 2009.

Keywords: biomass burning, Amazonia.

Index Terms: 0480 Biogeosciences: Remote sensing; 0315 Atmospheric Composition and Structure: Biosphere/atmosphere interactions (0426, 1610); 0305 Atmospheric Composition and Structure: Aerosols and particles (0345, 4801, 4906).

\section{Introduction}

[1] Recently, Koren et al. [2007] (hereinafter referred to as K2007) presented an analysis of biomass burning in Amazonia based on fire data from the NOAA-12 Advanced Very High Resolution Radiometer (AVHRR) and aerosol optical depth (AOD) data from the Terra Moderate Resolution Imaging Spectroradiometer (MODIS). In their study, they sought to identify trends in biomass burning in Amazonia and to explain the large reduction in fires observed during 2006. They concluded that a steady increase in biomass burning occurred throughout the region from 1998 through 2005 and that the major decline in 2006 was linked to the tri-national environmental policy implemented in Madre de Dios (Peru), Acre (Brazil), and Pando (Bolivia) (together known as the MAP region) in western Amazonia. Here we discuss potential problems with the K2007 AVHRR data set and with assumptions made in their analysis which draw the K2007 conclusions into question.

\section{Discussion}

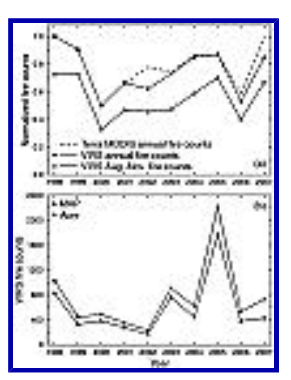

Figure 1. (a) Normalized TRMM VIRS annual and August-November fire counts for the K2007 study area covering $10^{\circ} \mathrm{N}-30^{\circ} \mathrm{S}$ and $30^{\circ} \mathrm{W}-$ $90^{\circ} \mathrm{W}$ (the shorter Terra MODIS fire time series is shown for comparison), and (b) annual TRMM VIRS fire counts for the MAP region and the state of Acre. 
[2] AVHRR active fire data made available from the National Institute for Space Research (INPE) in Brazil were used by K2007 as the primary source of information about the annual variation of fire activity in Amazonia. The authors used the 1998-2006 NOAA-12 AVHRR fire time series to substantiate their arguments, implicitly assuming that these data were produced consistently over the period analyzed. A comparison with the fire record obtained from the Tropical Rainfall Measuring Mission (TRMM) Visible and Infrared Scanner (VIRS) [Giglio et al., 2003] reveals a major discrepancy between the two time series: while VIRS shows highly elevated levels of fire activity in 1998 and 1999 (the highest in the past decade, in fact) the K2007 AVHRR time series shows relatively low levels of fire activity for this same time period (Figure 1a and compare Figure 4 of K2007).

[3] Severe drought conditions resulting from the onset of the $1998 \mathrm{El}$ Niño episode resulted in above average fire activity during that period, including a significant number of fire events which escaped into forest areas in eastern and southern Amazonia [Alencar et al., 2006]. While the VIRS time series captured this episode, the K2007 AVHRR time series seems to miss it entirely. Why is there such a large discrepancy between the two time series? Although we cannot provide a complete explanation at this time, based on recent discussions with INPE it is clear that the coverage area of AVHRR fire database gradually increased over time, particularly during the first few years of the last decade. Thus some portion of the apparent increase in AVHRR fire activity from 1998-2006 noted by K2007 is merely the result of the growing volume of AVHRR data being processed. Although the upward trend in the number of fires detected from 2001 through 2005 is confirmed with the VIRS time series, the K2007 depiction of a steady decadal trend in biomass burning is inadvertently misleading.

[4] Linking the seemingly drastic reduction in fire use in Amazonia during 2006 primarily to a change in environmental policy, K2007 employ a questionable assumption which ignores the complexity of the processes regulating fire use in the region [Cochrane, 2003; Nepstad et al., 2001]. Even if major progress in suppressing fire activity had been achieved within the trinational MAP region as K2007 suggest, the area contributes less than 5\% of the annual rates of deforestation and fire activity in Amazonia, compared to $60-70 \%$ for the combined states of Mato Grosso and Pará located in the southern and eastern parts of Brazilian Amazonia. These two states fall outside the scope of the MAP initiative (see auxiliary material Figure S1).

[5] Even if we consider the MAP region in isolation, the 4-fold drop in fire counts from 2005 to 2006 was preceded by a similar 4-fold increase in fire counts from 2004 to 2005 (Figure 1b). The extreme 2005 fire season in this region coincided with the most intense drought affecting western Amazonia in 34 years. In the specific case of Acre, the state government prohibited the use of vegetation fires by mid-August 2005 in response to the rapid increase in fire activity. Despite this regulation, fires continued to quickly spread, peaking in September and extending into October. From the annual fire statistics for Acre (Figure 1b) one can only conclude that the 2006 and 2007 fire seasons were comparable to the period prior to 2005 when normal climate conditions prevailed. Consequently, the effectiveness of the MAP initiative is not supported even within its own area of influence.

[6] In reality, fire detection statistics and anecdotal information suggests that the effectiveness of fire-use regulation tends to be confined to areas patrolled by law enforcement agents. For example, the Environmental Secretariat of Mato Grosso state passed a resolution in May 2006 prohibiting the use of vegetation fires during the period of 15 July through 15 September each year (i.e., the peak of the dry season in the region. (Secretaria de Estado do Meio Ambiente, Governo de Mato Grosso, http://www.sema.mt.gov.br, resolution 002, May 2006, 2008, available at http://www.sema.mt.gov.br/arquivos/resolucao\%2002\%20de\%2016_05_06.doc). However, approximately 10,000 and 17,600 Aqua MODIS fire counts were reported for that state by INPE during the fire prohibition period in 2006 and 2007, respectively (Instituto Nacional de Pesquisas Espaciais, BDQueimadas Monitoramento de Focos de Calor, 2008, available at http://www.dpi.inpe.br/proarco/bdqueimadas/). The relatively short duration of fire 
prohibition periods (usually a few weeks) is another factor which can lead to short-lived effects on fire statistics. It is therefore highly implausible that a basin-wide reduction in fire activity could result primarily from a change in governmental policies for Amazonia.

[7] Although K2007 acknowledged precipitation as a factor influencing fire activity in Amazonia, the authors understated its importance in their analysis. While K2007 report the late arrival of positive precipitation anomaly conditions during 2006, our assessment of precipitation in the region demonstrates that widespread positive anomalies affected the eastern and southern parts of Amazonia during September 2006 (auxiliary material Figure S2).

[8] Rainfall patterns in Amazonia changed from wet to dryer conditions during the 1999-2005 period, followed by another wet year in 2006. Each year from 2001-2005 the month of September consistently produced the largest numbers of fire counts. The areas under the influence of the positive precipitation anomaly in 2006 included parts of Mato Grosso and Pará, which as mentioned above account for the largest percentage of deforestation rates and fire activity in all Amazonia. K2007's reference to Acre state in Brazilian Amazonia is also somewhat misleading as Acre contributes less than $2 \%$ of all fire counts observed in Brazilian Amazonia during 2000-2005; fire statistics for this state are therefore not necessarily representative of Amazonian conditions. At least two major effects may result from aboveaverage precipitation: (1) increased fuel moisture will reduce fire ignition and spread [Uhl et al., 1988]; and (2) increased wet deposition should reduce the lifetime of suspended aerosol particles and their transport downwind [Freitas et al., 2005], thus influencing the concentration of such particles as depicted in the AOD product used by K2007.

\section{Conclusion}

[9] Biomass burning is a notoriously important phenomenon contributing to the accelerated depletion of Amazonia's tropical forests. Deforestation and fires are known to represent the two primary processes leading to the partial or full conversion of hundreds of thousands of hectares of Amazonian forests into croplands or pasture each year [Morton et al., 2006]. Forest depletion has reached alarming rates over the past two decades, followed in many periods by accelerated fire use as recorded by satellite-based fire products. Consequently, the need for effective environmental policies to suppress fire activity in the region is urgent and the societal response to the problem of vegetation fires can be seen in the recent initiatives promoting enhanced fireuse regulation [Brown et al., 2006]. Nonetheless, the complexities involving the social and physical drivers of fire activity in the region require careful consideration of those factors in order to assure proper assessment of this important issue.

[10] Despite being active supporters of initiatives such as MAP, it is known that (i) the use of fire is embedded in the culture of most Amazonian residents, and changing that culture may require years of investment in education and outreach in conjunction with viable alternatives for sustainable development; (ii) the pressure exerted by the agents promoting deforestation, fire use, and forest degradation overwhelms all regulatory instruments currently in use by the state and federal governments to thwart the almost chaotic situation in many parts of the region. A basin-wide comprehensive policy change may be required to alter current land use practices in the region and to promote steady reduction of deforestation and biomass burning activities.

[11] Based on the fire-use history in Amazonia during the past decade, it is plausible that cyclic oscillations, including strong inter-annual variations in fire activity such as seen in 1998-2000, 2005-2006, and 2006-2007, are determined in great part by climate anomalies fueled by social and economic factors. Hence we suggest that the conclusions drawn by K2007, particularly in light of the short duration of the satellite time series used in their study, be reconsidered to avoid a misperception of the problem of vegetation fires in Amazonia. 
Comment on "Reversal of trend of biomass burning in the Amazon" by Ilan Koren, L... Page 4 of 4

Citation: Schroeder, W., L. Giglio, and J. A. Aravéquia (2009), Comment on "Reversal of trend of biomass burning in the Amazon” by Ilan Koren, Lorraine A. Remer, and Karla Longo, Geophys. Res. Lett., 36, L03806, doi:10.1029/2008GL035659.

Copyright 2009 by the American Geophysical Union. 